\title{
Kidney Regeneration with Stem Cells: An Overview
}

\author{
Takashi Yokoo \\ Division of Nephrology and Hypertension, Department of Internal Medicine, The Jikei University School of Medicine, \\ Tokyo, Japan
}

\section{Key Words}

Regeneration · Stem cells · Dialysis

\begin{abstract}
Background: Kidney regeneration is currently gaining considerable attention in place of kidney dialysis as the ultimate therapeutic strategy for renal failure. However, because of anatomical complications, the kidney is believed to be the hardest organ to regenerate. Such a complicated organ is virtually impossible to imagine being completely rebuilt de novo from stem cells. Nevertheless, several research groups are attempting this large challenge. Summary: There are 4 major strategies for de novo kidney regeneration from stem cells. These strategies include the use of: (i) a decellularized cadaveric scaffold, (ii) blastocyst decomplementation, (iii) a nephrogenic niche for growing a xeno-embyro, and (iv) selfassembly potential. All of these strategies may be applicable in the clinical setting, but a substantial preparation period appears to be required. Key Messages: Although many outstanding problems remain for kidney regeneration, including ethical issues and the formation of chimeric structures, trials provide hope for dialysis patients and kidney regeneration is expected to be a reality in the future.
\end{abstract}

(c) 2014 S. Karger AG, Basel

\section{KARGER}

E-Mail karger@karger.com

www.karger.com/nee

\section{Introduction}

The kidney retains the potential to regenerate if the damage is not too severe and the kidney structure remains intact. However, in cases of irreversible damage to the kidney, as can occur with long-term dialysis, the property of self-renewal is totally lost. Therefore, any application of regenerative medicine in dialysis patients will require de novo development of an entire functional kidney.

In terms of a functional whole kidney, Chan et al. [1] reported the first attempt to develop a whole functional renal unit by forming a transplantable pronephros from animal caps in Xenopus. Transplantation of this pronephros-like unit at least partially corrected the edema in bilaterally nephrectomized tadpoles, and they survived for up to 1 month. To the best of our knowledge, this study is the only one in which a transplantable functional whole kidney unit has been developed de novo. However, the pronephros structure formed in this study was too primitive for any clinical application in humans. Since then, many attempts have been made worldwide to regenerate whole kidneys de novo (applicable in mammals) from stem cells. 


\section{Whole Kidney Reconstruction Using a Decellularized Cadaveric Scaffold}

It has been reported that decellularized cadaveric scaffolds can provide a niche for stem cells to differentiate into whole organs. This strategy was used by Ott et al. [2] to successfully develop a functional artificial rat heart. A whole-heart scaffold with an intact three-dimensional (3D) geometry and vasculature was created via coronary perfusion with detergents into the cadaveric heart, followed by repopulation with neonatal cardiac cells or rat aortic endothelial cells [2]. The injected neonatal cardiac cells formed a contractile myocardium, which performed the stroke function. This strategy has also been employed to develop transplantable liver and lungs using mature hepatocyte and alveolar epithelial cells, respectively $[3,4]$. Several attempts were made to use this technique for kidney regeneration. These attempts revealed that infused pluripotent stem cells were localized to the vasculature and glomeruli, with subsequent migration into the tubules, but it was difficult to acquire kidney function $[5,6]$. However, recently the same group, which successfully used the method described above to generate heart and lungs, reported successful whole kidney regeneration, which may produce urine after transplantation [7]. Notably, they used well-differentiated human umbilical vein endothelial cells instead of pluripotent stem cells, and use of only a scaffold selectively provided them with a niche for differentiated diversion of the renal and vascular residential cells in the right area. Although it is not clear how the infused cells differentiate and orchestrate into nephrons with vasculature to produce urine, this technique could be a solution for the shortage of donor organs.

\section{Blastocyst Complementation}

Injection of normal embryonic stem (ES) cells into the blastocysts of recombination-activating gene 2-deficient mice, which have no mature B or T lymphocytes, generates somatic chimeras with ES cell-derived mature B and T cells [8]. This 'blastocyst complementation' system was recently applied to reconstruction of the entire organ. Kobayashi et al. [9] recently reported successful regeneration of a rat pancreas in the mouse via an interspecific blastocyst injection of induced pluripotent stem (iPS) cells. They injected rat iPS cells into Pdx-1-/- (pancreatogenesis-disabled) mouse blastocysts and found that the newborn chimeras of the rat and mouse processed an almost entirely iPS-derived pancreas. This success proves

Kidney Regeneration with Stem Cells: An Overview that when an empty developmental niche for an organ is provided, iPS cell-derived cellular progeny can occupy that niche and compensate developmentally for the missing contents of the niche. This forms a complicated organ composed almost entirely of cells derived from donor iPS cells, even if the blastocyte complementation involves different species. That research group recently generated a $\mathrm{Pdx}-1-/-$ pig and succeeded in generating a bigger pancreas using this technique [8]. These successful findings suggest that human-scale organs could theoretically be generated de novo.

This technique was recently applied to whole kidney reconstruction [10]. Following injection of mouse iPS cells into the blastocysts of Sall1-null mice, which lack both kidneys, most of the metanephroi consisted of iPS cell-derived differentiated cells. However, they could not obtain the infant in livestock after this manipulation for an unknown reason [11], suggesting that there is another problem to solve in this system for kidney regeneration. However, these findings strongly suggest that blastocyst complementation is a most promising strategy for regeneration of the kidney. These systems are not available for clinical use at this time because it is not possible to generate vascular and nervous systems. In addition, important ethical issues with the manipulation of blastocysts with iPS cells remain unresolved $[12,13]$. Nonetheless, this success highlights the rationale that the eventual clinical application of kidney regeneration must depend on developmental programming.

\section{Use of a Nephrogenic Niche for Growing Xeno-Embryos (Organogenic Niche Method)}

Regeneration of a whole functional kidney using a developing heterozoic embryo as an 'organ factory' has been attempted. This is based on the concept of 'borrowing' the developing program of a growing xeno-embryo by applying stem cells at the niche of organogenesis. During the development of the metanephros, the metanephric mesenchyme initially forms from the caudal portion of the nephrogenic cord and secretes glial cell line-derived neurotrophic factor (GDNF). This process induces the nearby wolffian duct to produce a ureteric bud. Researchers microinjected GDNF-expressing human mesenchymal stem cells (hMSCs) into the site of budding following this process [14]. The recipient embryo was grown in a whole embryo culture system, and the formed metanephros was developed in organ culture. Viral-free manipulation can also be performed using a thermoreversible GDNF poly- 


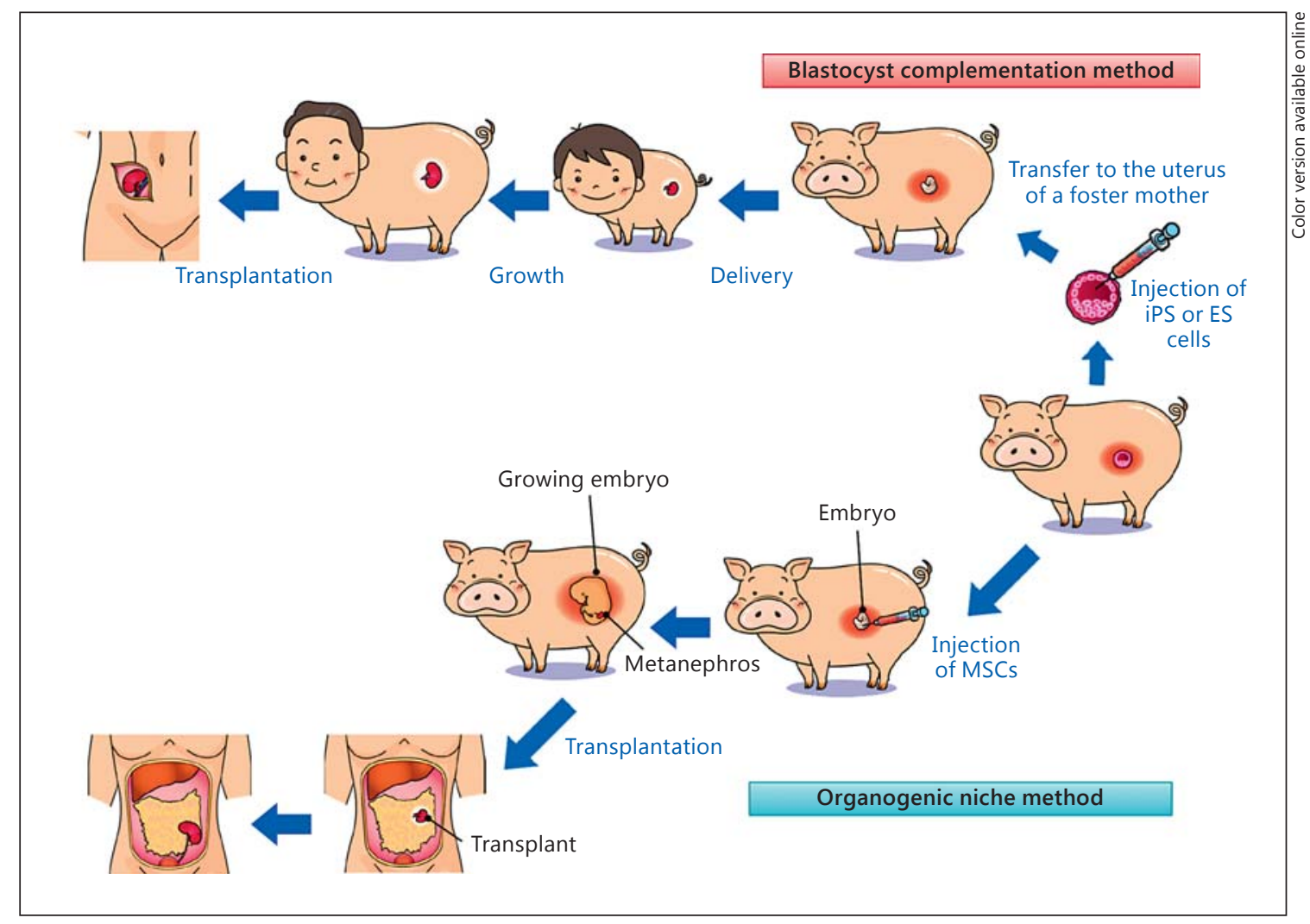

Fig. 1. Flow diagram of the blastocyst complementation and organogenic niche methods.

mer [15]. As a result, donor hMSCs were integrated into the rudimentary metanephros and morphologically differentiated to tubular epithelial cells, interstitial cells, and glomerular epithelial cells [14]. Researchers then transplanted the developed metanephros into the omentum to allow vascular integration from the recipient to form a functional nephron. As a result, an hMSC-derived 'neokidney' was generated, which contained a human nephron and the vasculature from the host [16]. In addition, the neokidney produced urine that showed higher concentrations of urea nitrogen and creatinine than the sera of the recipient. This finding suggested that the neokidney that developed in the omentum was capable of producing urine by filtering the recipient's blood [16]. Furthermore, the hMSC-derived neokidney secreted human erythropoietin and its production was stimulated by the induction of anemia in the host animal [17]. This finding indicated that this system preserves the normal physiological regulation of erythropoietin levels. However, the current system may not reconstruct derivatives of the ureteric bud. Therefore, to determine whether MSCs can differentiate into the ureteric bud progenitor using chick embryos, hMSCs expressing Pax2 were injected into the chicken ureteric bud progenitor region [18]. As a result, they migrated caudally with the elongating wolffian duct and were integrated into the wolffian duct epithelia and then expressed LIM1. This finding showed that they can differentiate into wolffian duct cells under the influence of local xeno-signals [18]. These results suggest that a whole kidney may be rebuilt by transplanting hMSCs at a suitable time and location to regenerate derivatives of the metanephric mesenchyme and ureteric bud.

Based on our successful findings, we are currently investigating the possibility of experimenting on a larger animal (i.e. the pig) because the porcine kidney has almost the same volume as the human kidney. The ultimate size of the developed metanephros appears to be imprinted during the early stages of development in the host embryo. This possibility is supported by the finding that the metanephroi of larger animals transplanted into the omenta of smaller hosts develop into organs with a larger 
volume (diameter and weight) compared to that of a normal host kidney [19]. Hopefully, this system will facilitate the development of larger organs that are more suitable for use in humans.

\section{Self-Assembly Potential of Stem Cells}

Some researchers have suggested that pluripotent stem cells have the potential to differentiate into mature cells and self-assemble into tissues or organs, and they have conducted investigations using pluripotent stem cells to generate mature cells in vitro. The autonomous formation of 3D tissue similar to the adenohypophysis [20], the optic cap [21], and intestinal tissue [22] structures using a 3D ES cell culture system from ES cells has been demonstrated. This approach could substantially reduce the complexity of organogenesis for therapeutic regeneration. To regenerate renal cells using such an approach, ES or iPS cells have to be differentiated into first intermediate mesoderm and then renal progenitors, followed by several types of renal cells. With regard to kidney regeneration, Osafune et al. [23] demonstrated that a single multipotent progenitor cell from an embryonic mouse kidney, which highly expresses Sall1, could differentiate into several types of renal cells, including glomerular podocytes and renal tubular epithelia, and eventually reconstruct a 3D kidney structure. Another recent study reported single-cell suspensions from embryonic kidney reaggregating to form organotypic renal structures [24]. During development, the kidney is derived from the intermediate mesoderm, one of the early germ layers. Intermediate mesoderm cells then differentiate into renal progenitors, followed by several types of renal cells. Therefore, if ES or iPS cells can differentiate first into intermediate mesoderm and then into renal progenitors, it is feasible that all types of renal cells could be generated using pluripotent stem cells. Osafune et al. [23] have established methods for differentiating human
iPSCs into intermediate mesoderm cells using a combination treatment of growth factors [25]. These cells express intermediate mesoderm marker genes and could mature into multiple cell types, including those found in intermediate mesoderm-derivative organs such as the kidney, gonads, and adrenal cortex. These investigations suggest that, if these intermediate mesoderm cells can differentiate into renal progenitor cells, a 3D kidney structure may be constructed from pluripotent stem cells. The means for successfully regenerating a functional vascular system between the regenerated kidney and the recipient remain unknown. In addition, the in vivo function of a regenerated kidney is unclear. However, further advances in developmental biology may resolve these issues and allow in vitro whole kidney regeneration.

\section{Conclusion}

This article summarizes the latest investigations on the use of stem cells to regenerate a functional whole kidney de novo. Despite many biological and technical advances in kidney regeneration, reconstruction of a fully functional kidney remains out of reach, and many problems are still unsolved. Use of heterologous tissue, such as xeno-metanephroi and xeno-blastocysts, raises ethical problems, while methods for reliably differentiating ESCs/iPSCs into kidneys in vitro have not been completely established. Methods for ensuring the function of regenerated renal tissue to produce urine and erythropoietin still need to be developed. However, continued efforts in stem cell and developmental biology will hopefully resolve these issues, leading to the development of new treatment strategies to reconstruct a whole kidney with adequate renal function. We believe that such efforts will come to fruition and it will be possible to regenerate a functional kidney in the future.

\section{References}

1 Chan T, Ariizumi T, Asashima M: A model system for organ engineering: transplantation of in vitro induced embryonic kidney. Naturwissenschaften 1999;86:224-227.

- 2 Ott HC, Mattheisen S, Goh SK, Vlack LD, Kren SM, Netoff TI, Taylor DA: Perfusiondecellularized matrix: using nature's platform to engineer a bioartificial heart. Nat Med 2008;14:213-221.

-3 Uygun BE, Soto-Gutierrez, A, Yagi H, Izamis ML, Guzzardi MA, Shulman C, Milwid J, Ko- bayashi N, Tiles A, Berthiaume F, Hertl M, Nahmias Y, Yarmush ML, Uygun K: Organ reengineering through development of a transplantable recellularized liver graft using decellularized liver matrix. Nat Med 2010;16: 814-820.

-4 Ott HC, Clippinger B, Conrad C, Schuetz C, Pomerantseva I, Ikonomou L, Kotton D, Cacanti JP: Regeneration and orthotopic transplantation of a bioartificial lung. Nat Med 2010;16:927-933.
Kidney Regeneration with Stem Cells: An Overview
Nephron Exp Nephrol 2014;126:54-58 DOI: $10.1159 / 000360662$ 
5 Ross EA, Williams MJ, Hamazaki T, Terada N, Clapp WL, Adin C, Ellison GW, Jorgensen M, Matich CD: Embryonic stem cells proliferate and differentiate when seeded into kidney scaffolds. J Am Soc Nephrol 2009;20:2338-2347.

-6 Orlando G, Farney AC, Iskandar SS, Mirmalek-Sani SH, Sullivan DC, Moran E, AbouShwareb T, De Coppi P, Wood KJ, Stratta RJ, Atala A, Yoo JJ, Soker S: Production and implantation of renal extracellular matrix scaffolds from porcine kidneys as a platform for renal bioengineering investigations. Ann Surg 2012;256:363-370.

-7 Song JJ, Guyette JP, Gilpin SE, Gonzalez G, Vacanti JP, Ott HC: Regeneration and experimental orthotopic transplantation of a bioengineered kidney. Nat Med 2013;19:646651.

8 Chen J, Lansford R, Stewart V, Young F, Alt FW: RAG-2-deficient blastocyst complementation: an assay of gene function in lymphocyte development. Proc Natl Acad Sci USA 1993;90:4528-4532.

-9 Kobayashi T, Yamaguchi T, Hamanaka S, Kato-Itoh M, Yamazaki Y, Ibata M, Sato H, Lee YS, Usui J, Knisely AS, Hirabayashi M, Nakauchi $\mathrm{H}$ : Generation of rat pancreas in mouse by interspecific blastocyst injection of pluripotent stem cells. Cell 2010;142:787799.

-10 Matsunari H, Nagashima H, Watanabe M, Umeyama K, Nakano K, Nagaya M, Kobayashi T, Yamaguchi T, Sumazaki R, Herzenberg LS, Nakauchi H: Blastocyst complementation generates exogenic pancreas in vivo in apancreatic cloned pigs. Proc Natl Acad Sci USA 2013;110:4557-4562.
1 Usui J, Kobayashi T, Yamaguchi T, Knisely AS, Nishinakamura R, Nakauchi H: Generation of kidney from pluripotent stem cells via blastocyst complementation. Am J Pathol 2012;180:2417-2426.

12 Normile D: Chimeric embryos may soon get their day in the sun. Science 2013;340:15091510.

13 Syranoski D: Japan to offer fast-track approval path for stem cell therapies. Nat Med 2013; 19:510.

14 Yokoo T, Ohashi T, Shen JS, Sakurai K, Miyazaki $Y$, Utsunomiya $Y$, Takahashi M, Terada Y, Eto Y, Kawamura T, Osumi N, Hosoya T: Human mesenchymal stem cells in rodent whole-embryo culture are reprogrammed to contribute to kidney tissue. Proc Natl Acad Sci USA 2005;102:3296-3300.

15 Gheisari Y, Yokoo T, Matsumoto K, Fukui A, Sugimoto N, Ohashi T, Kawamura T, Hosoya T, Kobayashi E: A thermoreversible polymer mediates controlled release of GDNF to enhance kidney regeneration. Artif Organs 2010;34:317-331.

16 Yokoo T, Fukui A, Ohashi T, Miyazaki Y, Utsunomiya $\mathrm{Y}$, Kawamura T, Hosoya T, Okabe M, Kobayashi E: Xenobiotic kidney organogenesis from human mesenchymal stem cells using a growing rodent embryo. J Am Soc Nephrol 2006;17:1026-1034.

17 Yokoo T, Fukui A, Matsumoto K, Ohashi T, Sado Y, Suzuki H, Kawamura T, Okabe M, Hosoya T, Kobayashi E: Generation of transplantable erythropoietin-producer derived from human mesenchymal stem cells. Transplantation 2008;85:1654-1658.

18 Fukui A, Yokoo T, Matsumoto K, Kawamura T, Hosoya T, Okabe M: Integration of human mesenchymal stem cells into the Wolffian duct in chicken embryo. Biochem Biophys Res Commun 2009;385:330-335.
19 Hammerman MR: Renal organogenesis from transplanted metanephric primordia. J Am Soc Nephrol 2004;15:1126-1132.

20 Suga $\mathrm{H}$, Kadoshima T, Minaguchi M, Ohgushi M, Soen M, Nakano T, Takata N, Wataya T, Muguruma K, Miyoshi H, Yonemura S, Oiso Y, Sasai Y: Self-formation of functional adenohypophysis in three-dimensional culture. Nature 2011;480:57-62.

21 Eiraku M, Takata N, Ishibashi H, Kawada M, Sakakura E, Okuda S, Sekiguchi K, Adachi T, Sasai Y: Self-organizing optic-cup morphogenesis in three-dimensional culture. Nature 2011;472:51-56.

22 Spence JR, Mayhew CN, Rankin SA, Kuhar MF, Vallance JE, Tolle K, Hoskins EE, Kalinichenko VV, Wells SI, Zorn AM, Shroyer NF, Wells JM: Directed differentiation of human pluripotent stem cells into intestinal tissue in vitro. Nature 2011;470:105-109.

23 Osafune K, Takasato M, Kispert A, Asashima M, Nishinakamura R: Identification of multipotent progenitors in the embryonic mouse kidney by a novel colony-forming assay. Development 2006;133:151-161.

24 Unbekandt M, Davies JA: Dissociation of embryonic kidneys followed by reaggregation allows the formation of renal tissues. Kidney Int 2010;77:407-416.

25 Mae S, Shono A, Shiota F, Yasuno T, Kajiwara M, Gotoda-Nishimura N, Arai S, Sato-Otubo A, Toyoda T, Takahashi K, Nakayama N, Cowan CA, Aoi T, Ogawa S, McMahon AP, Yamanaka S, Osafune K: Monitoring and robust induction of nephrogenic intermediate mesoderm from human pluripotent stem cells. Nat Commun 2013;4:1367. 